\title{
Reconstruction of superior mesenteric artery by prostheses placement in a case of chronic mesenteric ischemia: A case report and literature review
}

\author{
VLADISLAV BRASOVEANU ${ }^{1,2}$, DRAGOS ROMANESCU ${ }^{3}$, CAMELIA DIACONU $^{4,5}$, LAURA ILIESCU $^{4,6}$, \\ OVIDIU BRATU ${ }^{7,8}$, CORNEL SAVU ${ }^{9,10}$, CARMEN SAVU ${ }^{11}$, ADRIAN NEACSU ${ }^{12,13}$, \\ BOGDAN SOCEA $^{14}$, IRINA BALESCU ${ }^{15}$ and NICOLAE BACALBASA ${ }^{12,16,17}$
}

${ }^{1}$ Department of Surgery, 'Dan Setlacec' Center of Gastrointestinal Diseases and Liver Transplantation, Fundeni Clinical Institute, 022328 Bucharest; ${ }^{2}$ Department of Surgery, 'Titu Maiorescu' University of Medicine and Pharmacy, 040441 Bucharest; ${ }^{3}$ Department of Surgery, Sanador Medical Center, 011038 Bucharest; ${ }^{4}$ Department of Internal Medicine, 'Carol Davila' University of Medicine and Pharmacy, 020021 Bucharest; ${ }^{5}$ Department of Internal Medicine, Clinical Emergency Hospital Bucharest, 105402 Bucharest;

${ }^{6}$ Department of Internal Medicine, Fundeni Clinical Institute, 022328 Bucharest; ${ }^{7}$ Department of Urology,

'Carol Davila' University of Medicine and Pharmacy, 020021 Bucharest; ${ }^{8}$ Department of Urology, 'Carol Davila' Central Military Emergency University Hospital, 010825 Bucharest; ${ }^{9}$ Department of Thoracic Surgery,

'Carol Davila' University of Medicine and Pharmacy, 020021 Bucharest; ${ }^{10}$ Department of Thoracic Surgery, 'Marius Nasta' Institute of Pneumonology, 050159 Bucharest; ${ }^{11}$ Department of Anesthesiology, Fundeni Clinical Institute, 022328 Bucharest; ${ }^{12}$ Department of Obstetrics and Gynecology, 'Carol Davila' University of Medicine and Pharmacy, 020021 Bucharest; ${ }^{13}$ Department of Obstetrics and Gynecology, 'Sf. Ioan' Clinical Emergency Hospital,

042122 Bucharest; ${ }^{14}$ Department of Surgery, 'Sf. Pantelimon' Clinical Hospital, 021659 Bucharest;

${ }^{15}$ Department of Surgery, 'Ponderas' Academic Hospital, 021188 Bucharest; ${ }^{16}$ Department of Visceral Surgery, Center of Excellence in Translational Medicine, Fundeni Clinical Institute, 022328 Bucharest;

${ }^{17}$ Department of Obstetrics and Gynecology, 'I. Cantacuzino' Clinical Hospital, 030167 Bucharest, Romania

Received June 3, 2020; Accepted July 3, 2020

DOI: $10.3892 / \mathrm{etm} .2020 .9119$

\begin{abstract}
Although superior mesenteric artery stenosis is a relatively common situation, it is rarely symptomatic due to the fact that in a significant number of cases an adequate collateral circulation exists. The aim of this study is to report a case in which arterial reconstruction was needed due to the absence of such a patent collateral circulation. The 47-year-old patient was investigated for chronic postprandial pain and was diagnosed with superior mesenteric artery stenosis. Percutaneous treatment was the initial option of choice but the patient rapidly became symptomatic again. Therefore surgery was performed,
\end{abstract}

Correspondence to: Professor Nicolae Bacalbasa, Department of Obstetrics and Gynecology, 'I. Cantacuzino' Clinical Hospital, 5-7 Ion Movilă Street, 030167 Bucharest, Romania

E-mail: nicolae_bacalbasa@yahoo.ro

Abbreviation: PTFE, polytetrafluoroethylene

Key words: superior mesenteric artery, stenosis, percutaneous treatment, prosthesis, reconstruction the segment of arterial stenosis was resected and the arterial continuity was re-established by using a synthetic prosthesis. The postoperative outcome was uneventful, the patient was discharged in the seventh postoperative day under anticoagulant therapy. In conclusion, superior mesenteric artery reconstruction by using a synthetic prosthesis can be useful in cases presenting chronic mesenteric ischemia and failure of percutaneous treatment.

\section{Introduction}

Although superior mesenteric artery stenosis represents a relatively frequent pathological condition with increasing prevalence with age, it rarely becomes symptomatic due to the fact that most often the presence of an adequate collateral circulation will prevent the apparition of chronic mesenteric ischemia (1-3). Symptomatic chronic mesenteric ischemia was initially described at the end of the eighteen century $(4,5)$, while the first surgical approach was described more than 50 years ago $(6,7)$. At present, the main options of treatment are represented by endovascular and open approach. While endovascular surgery is usually less invasive than open surgery, being the initial option of choice in a significant number of cases, its results might be less durable and open approach might be needed if 
symptoms reappear (8-10). The present study report a case of a 47-year-old man in whom re-appearance of symptoms after endovascular treatment imposed performing an open approach. Approval for the publication of the data was obtained from the Medical Ethics Committee of the Fundeni Clinical Institute (no. 28/2020; Bucharest, Romania) and written informed consent was obtained from the patient prior to the study.

\section{Case report}

The 47-year-old male with no significant health history was investigated for postprandial abdominal pain, with onset within the first hours after meal which had been observed by the patient three months previously and progressively accentuated. According to the patient's description, the onset of the pain was usually within the first hour after ending the meal, while the duration was of one to one and a half hours. Moreover, a weight loss of $10 \mathrm{~kg}$ in the last three months was also associated and was rather explained by the patient's fear of eating in order not to induce the development of the abdominal pain. The patient was further submitted to an upper abdominal gastroscopy, which demonstrated the presence of diffuse chronic gastritis, and to a lower digestive endoscopy which failed to demonstrate any cause for this symptomatology. When testing which type of nutrients induce this type of pain, the gastroenterologist observed that the peak of intensity was induced by the administration of fatty nutrients.

The computed tomographic angiography demonstrated the presence of a stenosis of the superior mesenteric artery at $0.5 \mathrm{~cm}$ from the aortic origin, measuring $1 \mathrm{~cm}$ in length, which exceeded $75 \%$ of the mesenteric caliber with no other pathological aspects. Due to the fact that the patient was intensely symptomatic and due to the presence of these radiological findings, he was initially submitted to a percutaneous dilatation by using an endovascular balloon; although initially the postprandial pain diminished, the symptoms reappeared within the first six months, so the patient was considered as a candidate for surgery. The superior mesenteric artery was dissected and the stenotic segment was resected; the continuity of the superior mesenteric artery was re-established by placing a Dacron prosthesis which was implanted at the level of the abdominal aorta and was anastomosed with the stump of the mesenteric artery (Figs. 1-3). An intraoperative Doppler ultrasound was performed and demonstrated the presence of a good blood flow at the level of the synthetic patch. Intraoperatively, heparin treatment was initiated, and replaced during the early postoperative period with low molecular weight heparin. The postoperative course was uneventful the patient being discharged in the seventh postoperative day.

\section{Discussion}

Superior mesenteric artery stenosis reports an increasing prevalence with age, from $<6 \%$ in patients younger than 40 years of age to $>65 \%$ among patients $>75$ years of age, these data being comparable to those reported so far for other peripheral vascular diseases, such as coronary of cerebral ones $(11,12)$. However, while in cases presenting cardiac or cerebrovascular disease the development of ischemic events is a common eventuality being reported in up to $20 \%$ of cases, patients

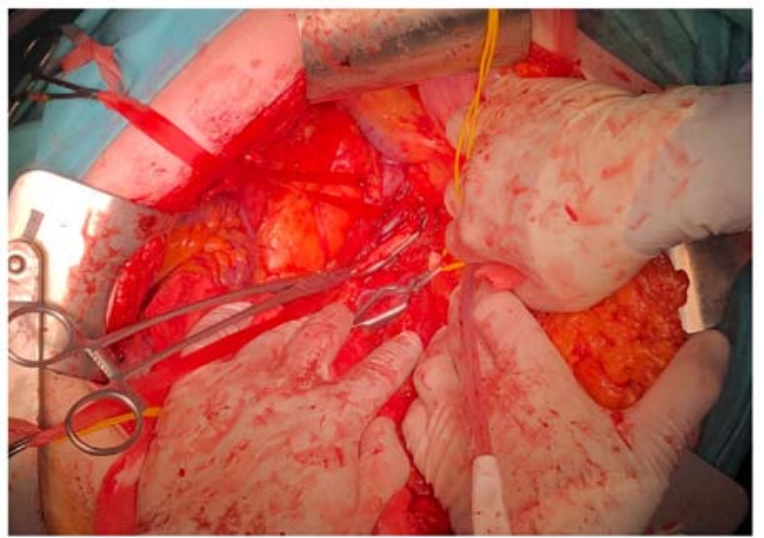

Figure 1. The aspect after identification and mobilization of the stenotic segment of the superior mesenteric artery.

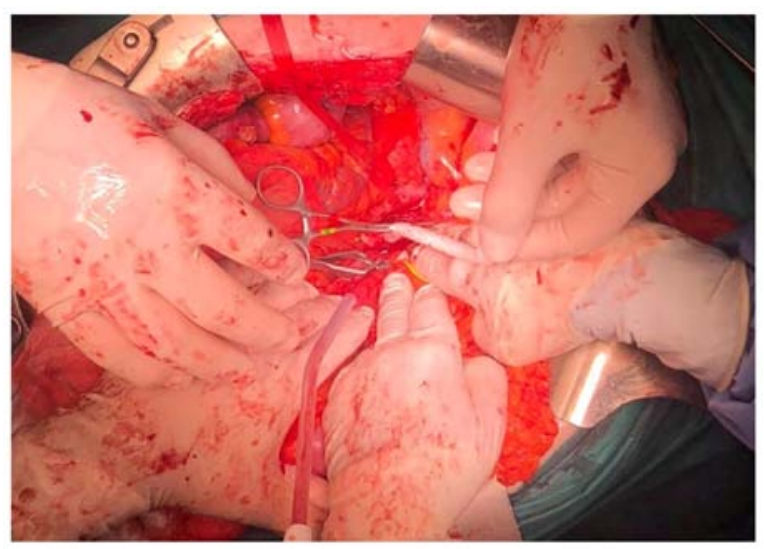

Figure 2. The aspect after resection of the stenotic segment; the continuity of the superior mesenteric artery to be re-established by placing the Dacron prosthesis.

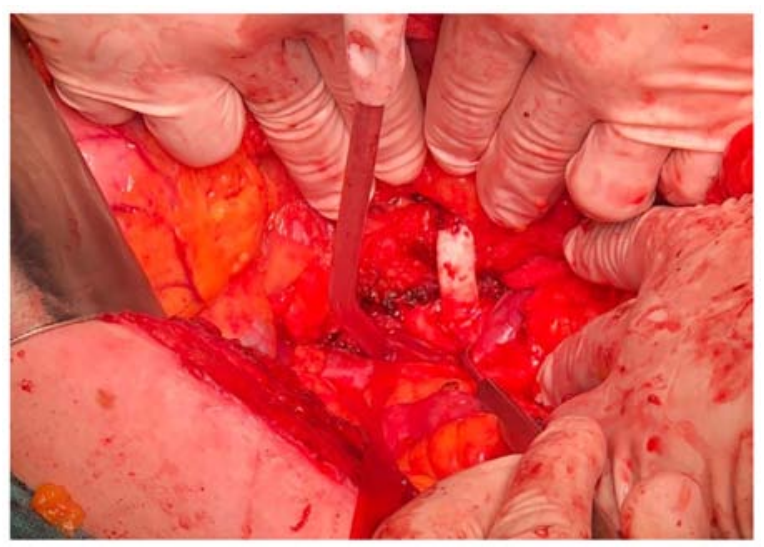

Figure 3. The final aspect after reconstruction of the superior mesenteric artery by Dacron prosthesis placement.

presenting superior mesenteric artery stenosis this situation in scarcer, being reported in up to $5 \%$ of cases. This fact is rather explained by the presence of a rich vascular anastomotic system which is developed at the mesenteric level and which provides a good collateral circulation as well as the delay in the appearance of clinical symptoms $(2,13)$. According to the study of Someya et al (14), the baseline flow at the level of 
superior mesenteric artery is $400 \mathrm{ml} / \mathrm{min}$ and increases up to $800 \mathrm{ml} / \mathrm{min}$ at $40 \mathrm{~min}$ after a meal, this increased value persists for up to $3 \mathrm{~h}$. In this respect it is evident why the presence of a significant decrease of the blood flow at this level in the absence of a patent collateral circulation will become intensely symptomatic and will impose performing the procedure of revascularization.

In the last decade, due to the improvement of the endovascular surgical techniques such patients are initially considered to be candidates for percutaneous endovascular approach of the stenotic segment, consisting of femoral or brachial artery puncture, performing an angiography and re-permeabilization of the affected segment by using a dilatation balloon or by placing a stent $(15,16)$. The main indication for such procedures is represented by the presence of symptomatic ischemia inducing weight loss and severe post-prandial pain $(17,18)$. Most often, patients who benefit from percutaneous endovascular procedures present close to the aortic emergence lesions, with low length of the stenotic segment; whereas, patients considered as candidates for the open approach are usually those presenting more distal and extended lesions as well as those who return with failure and re-appearance of symptoms (19). However, while in cases submitted to percutaneous endovascular treatment restenosis and the need of reintervention is a common event, open surgery provides a significantly better chance for obtaining a durable result (10).

In the last decade, due to the improvement of surgical techniques, reconstructive procedures have been successfully used in patients presenting both benign and malignant abdominal conditions (20-27). In cases presenting benign conditions, different therapeutic strategies have been considered, such as replacement of the affected segment by placing an autologous or synthetic graft, or performing a bypass procedure $(20,23-25,27)$.

One of the first studies which came to demonstrate the effectiveness of reconstructive surgery for atherosclerotic occlusive disease affecting the superior mesenteric artery was conducted between 1975 and 1988 in Hospital Saint Joseph, Paris, France (28). In this study the authors introduced 103 patients with a mean age of 57.2 years, 20 of them being submitted to surgery for chronic, typical abdominal angina. During the early postoperative period, four cases experienced early re-occlusions, while after a median follow-up period of 69 months, further five cases presented recurrent mesenteric ischemia. Among the 103 cases there were 10 patients presenting isolated superior mesenteric artery stenosis, four of them being asymptomatic at the time of surgery, other four cases presenting nonspecific abdominal symptoms, one case presenting typical abdominal angina and another case being already submitted to surgery and intestinal resection for intestinal ischemia. Moreover, seven cases associated superior and inferior mesenteric artery stenosis, 24 cases associated superior mesenteric artery stenosis and celiac trunk stenosis, while the remaining 62 cases presented stenotic lesions in all the three vascular territories. Therefore, superior mesenteric artery reconstruction was performed as stand-alone procedure or in association with other vascular gestures in all 103 cases. Most often, revascularization was provided by performing a bypass; however, in 14 cases, resection and reconstruction was performed, in nine cases the superior mesenteric artery being reimplanted by using a prosthetic graft [in five patients a Dacron prosthesis was used while in the remaining four cases a polytetrafluoroethylene (PTFE), graft was the option of choice]. During the early postoperative period, none of these cases experienced any significant complications; during the late postoperative period five cases reported the appearance of ischemic complications; however, none of these cases had been submitted to reconstruction using Dacron prosthesis (28). Similar results were published three years later by the Danish study group conducted by Christensen et al (29) on 90 patients with atherosclerotic disease of the mesenteric arteries. Among these cases, there were 87 patients who necessitated the reconstruction of the superior mesenteric artery alone or in association with the celiac axis and/or with the inferior mesenteric artery. Superior mesenteric artery was reconstructed using a synthetic graft in 39 cases, Dacron prosthesis was placed in 36 cases and a PTFE graft was the option of choice in the remaining five cases. As for the postoperative outcomes, both early and long-term outcomes seemed to be significantly influenced by the indication for surgery, patients presenting acute intestinal ischemia at the time of surgery, reporting a significantly poorer outcome when compared with those presenting chronic lesions (29). As for the preferred techniques of arterial reconstruction by decade, Lejay et al (19) demonstrated that at the beginning of the 20th century prosthesis placement gained more popularity and provided better long-term outcomes when compared with other procedures such as bypasses; moreover, the authors underlined the fact that in a significant number of cases in which the endovascular treatment fails open surgery might provide a long lasting, durable revascularization of the affected territories.

In conclusion, although superior mesenteric artery stenosis is a common finding, situations in which revascularization for chronic abdominal pain is needed are rare; in such cases, the initial option of choice is represented by endovascular procedures such as balloon dilatation or stent placement. However, in certain cases presenting distal, prolonged areas of stenosis, percutaneous maneuvers might fail and open surgery might be needed. In such cases resection of the stenotic segment followed by prosthetic placement is demanding, but a very effective procedure.

\section{Acknowledgements}

Not applicable.

\section{Funding}

No funding was received.

\section{Availability of data and materials}

All data and images included and/or analyzed during the current study are available from the corresponding author on reasonable request.

\section{Authors' contributions}

VB and DR performed the surgical procedures. CD and LI were responsible for the preoperative investigations and made 
the diagnosis. NB, CoS, IB, OB, CaS, AN and BS performed the literature research regarding vascular techniques of re-permeabilization and reconstruction, and drafted the manuscript. NB reviewed the final version of the manuscript. All authors read and approved the final version of the manuscript.

\section{Ethics approval and consent to participate}

The Medical Ethics Committee of the Fundeni Clinical Institute (no. 28/2020; Bucharest, Romania) approved the study and gave consent for the publication of the data. Written consent was obtained from the patient regarding participation to the study. Data collection was carried out during hospitalization.

\section{Patient consent for publication}

Signed informed consent was obtained from the patient on 23.03.2020.

\section{Competing interests}

The authors declare that they have no competing interests.

\section{References}

1. Järvinen O, Laurikka J, Sisto T, Salenius JP and Tarkka MR: Atherosclerosis of the visceral arteries. Vasa 24: 9-14, 1995.

2. Wilson DB, Mostafavi K, Craven TE, Ayerdi J, Edwards MS and Hansen KJ: Clinical course of mesenteric artery stenosis in elderly Americans. Arch Intern Med 166: 2095-2100, 2006.

3. Kolkman JJ and Geelkerken RH: Diagnosis and treatment of chronic mesenteric ischemia: An update. Best Pract Res Clin Gastroenterol 31: 49-57, 2017.

4. Chienne J: Complete obliteration of celiac and mesenteric arteries. J Anat Physiol 3: 63-72, 1869.

5. Councilman WT: Three cases of occlusion of the superior mesenteric artery. Boston Med J 130: 410-411, 1894.

6. Derrick JR, Pollard HS and Moore RM: The pattern of arteriosclerotic narrowing of the celiac and superior mesenteric arteries. Ann Surg 149: 684-689, 1959.

7. Morris GC Jr, Crawford ES, Cooley DA and Debakey ME: Revascularization of the celiac and superior mesenteric arteries Arch Surg 84: 95-107, 1962.

8. Schermerhorn ML, Giles KA, Hamdan AD, Wyers MC and Pomposelli FB: Mesenteric revascularization: Management and outcomes in the United States, 1988-2006. J Vasc Surg 50: 341-348.e1, 2009.

9. Illuminati G, Caliò FG, D'Urso A, Papaspiropoulos V, Mancini P and Ceccanei G: The surgical treatment of chronic intestinal ischemia: Results of a recent series. Acta Chir Belg 104: 175-183, 2004.

10. Tallarita T, Oderich GS, Gloviczki P, Duncan AA, Kalra M, Cha S, Misra S and Bower TC: Patient survival after open and endovascular mesenteric revascularization for chronic mesenteric ischemia. J Vasc Surg 57: 747-755, 2013.

11. Selvin E and Erlinger TP: Prevalence of and risk factors for peripheral arterial disease in the United States: Results from the National Health and Nutrition Examination Survey, 1999-2000. Circulation 110: 738-743, 2004.

12. AbuRahma AF, Cook CC, Metz MJ, Wulu JT Jr and Bartolucci A: Natural history of carotid artery stenosis contralateral to endarterectomy: Results from two randomized prospective trials. J Vasc Surg 38: 1154-1161, 2003.
13. Thomas JH, Blake K, Pierce GE, Hermreck AS and Seigel E: The clinical course of asymptomatic mesenteric arterial stenosis. J Vasc Surg 27: 840-844, 1998.

14. Someya N, Endo MY, Fukuba Y and Hayashi N: Blood flow responses in celiac and superior mesenteric arteries in the initial phase of digestion. Am J Physiol Regul Integr Comp Physiol 294: R1790-R1796, 2008.

15. Resch TA, Acosta S and Sonesson B: Endovascular techniques in acute arterial mesenteric ischemia. Semin Vasc Surg 23: 29-35, 2010.

16. Vos J, de Vries JM and van Strijen ML: Endovascular management of chronic mesenteric ischemia. In: Endovascular Interventions. Dieter RS, Dieter JR and Dieter IIIRA (eds). Springer, New York, NY, pp429-434, 2014.

17. Loffroy R, Steinmetz E, Guiu B, Molin V, Kretz B, Gagnaire A, Bouchot O, Cercueil JP, Brenot R and Krausé D: Role for endovascular therapy in chronic mesenteric ischemia. Can J Gastroenterol 23: 365-373, 2009.

18. Loffroy R, Guiu B, Cercueil JP and Krausé D: Chronic mesenteric ischemia: Efficacy and outcome of endovascular therapy. Abdom Imaging 35: 306-314, 2010.

19. Lejay A, Georg Y, Tartaglia E, Creton O, Lucereau B, Thaveau F, Geny B and Chakfe N: Chronic mesenteric ischemia: 20 year experience of open surgical treatment. Eur J Vasc Endovasc Surg 49: 587-592, 2015.

20. Illuminati G, Pizzardi G, Calio' FG, Pasqua R, Masci F and Vietri F: Infrarenal aorta as the donor site for bypasses to the superior mesenteric artery for chronic mesenteric ischemia: A prospective clinical series of 24 patients. Surgery 162: 1080-1087, 2017.

21. Brasoveanu V, Anghel C, Barbu I, Pautov M, Ionescu MI, Motthor M, Balescu I, Dima S and Bacalbasa N: Pancreatoduodenectomy en bloc with portal and superior mesenteric artery resection - a case report and literature review. Anticancer Res 35: 1613-1618, 2015.

22. Braşoveanu V, Dumitraşcu T, Bacalbaşa N and Zamfir R: Splenic artery used for replaced common hepatic artery reconstruction during pancreatoduodenectomy - a case report. Chirurgia (Bucur) 104: 499-504, 2009.

23. Bacalbasa N, Brezean I, Anghel C, Barbu I, Pautov M, Balescu I and Brasoveanu V: Successful resection and vascular ligation of a large hepatic artery aneurysm - A case report and literature review. In Vivo 31: 979-982, 2017.

24. Bacalbasa N, Brezean I, Anghel C, Barbu I, Pautov M, Balescu I and Brasoveanu V: Management of a fulminant upper gastrointestinal bleeding exteriorized through hemobilia due to arteriobiliary fistula between the common bile duct and a right hepatic artery aneurysm - A Case Report. In Vivo 31: 983-989, 2017.

25. Bacalbasa N, Balescu I, Tanase A, Pautov M, Brezean I, Vilcu M and Brasoveanu V: Spleno-pancreatectomy en bloc with parcelar gastrectomy for splenic artery aneurysm - A case report and literature review. In Vivo 32: 915-919, 2018.

26. Brasoveanu V, Ionescu MI, Grigorie R, Mihaila M, Bacalbasa N, Dumitru R, Herlea V, Iorgescu A, Tomescu D and Popescu I: Living donor liver transplantation for unresectable liver adenomatosis associated with congenital absence of portal vein: A case report and literature review. Am J Case Rep 16: 637-644, 2015.

27. Bacalbasa N, Balescu I, Tanase A, Brezean I, Vilcu M and Brasoveanu V: Successful resection of a non-functional paraganglioma with celiac trunk invasion followed by common hepatic artery reimplantation - A case report and literature review. In Vivo 32: 911-914, 2018.

28. Cormier JM, Fichelle JM, Vennin J, Laurian C and Gigou F: Atherosclerotic occlusive disease of the superior mesenteric artery: Late results of reconstructive surgery. Ann Vasc Surg 5: 510-518, 1991.

29. Christensen MG, Lorentzen JE and Schroeder TV: Revascularisation of atherosclerotic mesenteric arteries: Experience in 90 consecutive patients. Eur J Vasc Surg 8: 297-302, 1994. 